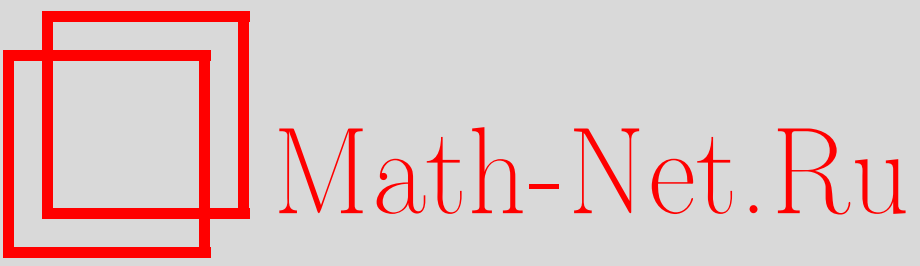

А. Ю. Вайнтроб, Алгеброиды Ли и гомологические векторные поля, УМН, 1997, том 52, выпуск 2, 161-162

DOI: https://doi.org/10.4213/rm831

Использование Общероссийского математического портала Math-Net.Ru подразумевает, что вы прочитали и согласны с пользовательским соглашением

http://www.mathnet.ru/rus/agreement

Параметры загрузки:

IP: 54.197 .130 .99

26 апреля 2023 г., 12:16:56 


\title{
АЛГЕБРОИДЫ ЛИ И ГОМОЛОГИЧЕСКИЕ ВЕКТОРНЫЕ ПОЛЯ
}

\author{
А. Ю. ВАЙнтрОБ
}

Понятие алгеброида Ли, введенное Дж. Прадине, является аналогом алгебры группы Ли для дифференцируемых группоидов. Алгеброиды Ли сочетают в себе свойства алгебр Ли и многообразий и находят применение в дифференциальной геометрии, симплектической геометрии и теории представлений (см. [1]-[3]). Цель этой заметки - продемонстрировать, что теория алгеброидов Ли является частным случаем теории гомологических векторных полей на супермногообразиях [4], [5], и указать на возможные применения такого подхода.

1. Определения. Векторное расслоение $A \rightarrow M$ со скобкой Ли [, ] на пространстве сечений $\Gamma(A)$ и гомоморфизмом расслоений $a: A \rightarrow T M$, назьваемым якорем, называется алгеброидом Ли, если $a$ - гомоморфизм алгебр Ли и $[x, f y]=(a(x) f) y+f \cdot[x, y]$ для $x, y \in A, f \in C^{\infty}(M)$.

Стандартные примеры алгеброидов Ли - это расслоения алгебр Ли $(a=0)$, касательное расслоение многообразия и кокасательное расслоение пуассонова многообразия.

Нечетное векторное поле $V$ на супермногообразии назьвается гомологическим векторныц полем, если $[V, V]=2 V^{2}=0$. Многие объекты из различных областей математики можно описывать и изучать в терминах гомологических полей (см. [4], [5]).

2. Теорема. Обозначим через $\mathscr{M}=\left(M, \Lambda^{\bullet} A^{*}\right)$ и $\mathscr{M}^{\prime}=\left(M, \Lambda^{\bullet} A\right)$ два супермногообразия, ассочиированных с расслоением $A \rightarrow M$. Следующие три класса обтектов находятся в естественном взаимно-однозначном соответствии:

(i) структуры алгеброида Ли на $A \rightarrow M$;

(ii) гомологические векторные поля степени 1 на $\mathscr{M}$;

(iii) нечетные линейнье пуассоновы структуры на $\mathscr{M}^{\prime}$.

ДокАЗАТЕЛЬСтво. В локальной системе координат $(x, \xi)$ на $\mathscr{M}$ (где $\left(x^{\alpha}\right)$ - координаты на $M$, а $\left(\xi^{i}\right)$ - локальный базис сечений $A^{*}$ ) любое векторное поле степени 1 имеет вид $V=\sum c_{i j}^{k} \xi^{i} \xi^{j} \partial_{\xi^{k}}+\sum a_{i}^{\alpha} \xi^{i} \partial_{x^{\alpha}}$, где $c$ и $a-$ функции, зависящие от $x^{\alpha}$. Обозначим через $\left(\varepsilon_{i}\right)$ локальный базис сечений $A$, дуальньй к $\left(\xi^{i}\right)$. Положим $a: A \rightarrow T M: a(X)=\sum f^{i} a_{i}^{\alpha} \partial_{x^{\alpha}}$ и $[X, Y]=\sum f^{i} g^{j} c_{i j}^{k} \varepsilon_{k}+\sum a(X)\left(g^{j}\right) \varepsilon_{j}-\sum a(Y)\left(f^{i}\right) \varepsilon_{i}$, где $X=\sum f^{i}(x) \varepsilon_{i}$ и $Y=\sum g^{i}(x) \varepsilon_{i} \in$ $\Gamma(A)$. Обратно, по скобке [, ] и отображению $a: A \rightarrow T M$ мы можем найти функции $c_{i j}^{k}$ и $a_{i}^{\alpha}$ и определить векторное поле $V$. Непосредственно проверяется, что пара $([], a$,$) задает структуру$ алгеброида Ли тогда и только тогда, когда $V$ - гомологическое векторное поле.

Каноническое спаривание между $A$ и $A^{*}$ преобразует $\xi^{i}$ в $\partial_{\varepsilon_{i}}, \partial_{\xi^{i}}$ в $\varepsilon_{i}$, и $V$ превращается в нечетное бивектроное поле $\pi=\sum c_{i j}^{k} \varepsilon_{k} \partial_{\varepsilon_{i}} \wedge \partial \varepsilon_{j}+\sum a_{i}^{\alpha} \partial_{\varepsilon_{i}} \wedge \partial_{x^{\alpha}}$ на $\mathscr{M}^{\prime}$. Из гомологической интерпретации пуассоновых структур [4] следует, что $\pi$ задает пуассонову структуру на $\mathscr{M}^{\prime}$ тогда и только тогда, когда $V$ - гомологическое поле. Это можно также проверить непосредственным вычислением. Скобка, задаваемая $\pi$, является нечетным вариантом линейной пуассоновой структуры на $A^{*}$, введенной Курантом [6].

3. Морфизмы. Ясно, что следует называть морфизмом алгеброидов Ли над одной базой $M$, в то время как общее определение, данное Аламейдой и Кумперой [7] (см. также [8]), нетривиально. Применять это определение трудно, и даже то, что композиция двух морфизмов является морфизмом, не очевидно. При переходе на язык гомологических векторных полей ситуация полностью меняется. Напомним, что морфизм двух векторных полей $V$ на $P$ и $W$ на $Q$ это отображение $h: P \rightarrow Q$ такое, что $h_{*}\left(V_{p}\right)=W_{h(p)}$ для любого $p \in P$.

Теорема. Пусть $V$ и $W$ - гомологические векторные поля на $\mathscr{M}$ u $\mathscr{N}$, соответствующие алгеброидам Ли $A \rightarrow M$ и $B \rightarrow N$. Морфизм расслоений $\varphi: A \rightarrow B, f: M \rightarrow N$ является морфизмом алгеброидов Ли тогда и только тогда, когда индуцированное отображение супермногообразий $\Phi: \mathcal{M} \rightarrow \mathcal{N}$ является морфизмом гомологических полей $V u W$. 
4. Модули над алгеброидами Ли. Напомним, что модуль над гомологическим векторным полем $V$ на супермногообразии $P$ - это расслоение $E \rightarrow P$ с плоской $V$-связностью $\nabla$ (т.е. линейньм отображением $\nabla: \Gamma(E) \rightarrow \Gamma(E)$ таким, что $\nabla(f e)=V(f) e+(-1)^{|f|} f \cdot \nabla e$ и $\left.\nabla^{2}=0\right)$. Это приводит к следующему определению. Модуль над алгеброидом Ли $A \rightarrow M$ - это однородное расслоение $E$ над $\mathscr{M}$ (т.е. $\mathbb{Z}$-градуированньй $C^{\infty}(\mathscr{M})$-модуль) с плоской $V$-связностью $\nabla$ степени 1. Пространство гомологий $H^{\bullet}(A ; E):=H(\nabla)=\operatorname{Ker}(\nabla) / \operatorname{Im}(\nabla)$ назьвается когомологиями $A$ с коэффициентами в $E$.

Представления алгеброида $A \rightarrow M$ в смысле Макензи [1] соответствуют специальному типу модулей, у которых $E=\pi^{*} F$, где $F$ - расслоение над $M$, а $\pi: \mathscr{M} \rightarrow M$-каноническая проекция.

Предложение. Расслоение $F \rightarrow M$ с линейным отображением $m: \Gamma(A) \otimes \Gamma(F) \rightarrow$ $\Gamma(F)$ является представлением алгеброида Ли $A \rightarrow M$ тогда и только тогда, когда индуцированное отображение $m^{*}: \Gamma(F) \rightarrow \Gamma(F) \otimes \Gamma\left(A^{*}\right)$, продолженное до $V$-связности $\nabla$ на $E=\pi^{*} F \rightarrow \mathscr{M}$, является $A$-модулем $\left(\right.$ т.е. $\left.\nabla^{2}=0\right)$.

5. Пример: тензорные модули. Для гомологического векторного поля $V$ на $\mathscr{M}$ пространство тензоров на $\mathscr{M}$ определенного типа является $V$-модулем с $\nabla=L_{V}$, производная Ли. В частности, если $V$ - гомологическое векторное поле, соответствующее алгеброиду Ли $A \rightarrow M$, мы получаем тензорные $A$-модули. За исключением тривиалшного случая, когда $a=0$ (т.е. когда $A$ - это расслоение алгебр Ли), произвольньй тензорньй модул не является представлением в смысле [1]. Тем не менее, к этому типу относятся такие важные модули, как присоединенньй $A d=T \mathscr{M}$, коприсоединенный $A d^{*}=\Omega^{1} \mathscr{M}$ и дуализирующий $\operatorname{Ber}=\operatorname{Vol}(\mathscr{M})$. Если $\mathscr{M}$ компактно, интегрирование задает невырожденное спаривание между $\operatorname{Vol}(\mathscr{M})$ и $C^{\infty}(\mathscr{M})$, совместимое с $\mathbb{Z}$-градуировкой. Оно индуцирует каноническую двойственность между $H^{\bullet}(A)$ и $H^{\bullet}(A ; \operatorname{Ber})$, обобщающую как классическую двойственность Пуанкаре, так и ее аналог для алгебр Ли.

6. Деформации. Как и в случае алгебр Ли, когомологии алгеброида Ли с коэффициентами в присоединенном модуле возникают при изучении деформаций. Результаты о деформациях гомологических векторных полей [5] дают следующую теорему. Tогда

(i) алгебра Ли группьи автоморфизмов $А$ изоморфна $H^{0}(A, A d)$;

(ii) пространство инфинитезимальных деформаций $A$ изоморфно $H^{1}(A, A d)$;

(iii) препятствия к продолжению деформачий принадлежат $H^{2}(A, A d)$;

(iv) если $\operatorname{dim} H^{1}(A, A d)=d<\infty u H^{2}(A, A d)=0$, то у алгеброида $A$ существует версальная деформачия с гладкой базой размерности $d$.

\section{СПИСОК ЛИТЕРАТУРЫ}

[1] Mackenzie K. Lie grupoids and Lie algebroids in differential geometry // London Math. Soc. Lect. Note Ser. V. 124. Cambridge: Cambridge Univ. Press, 1987. [2] Coste A., Dazord P., Weinstein A. // Publ. Dép. Math. Univ. Claude-Bernard, Lyon. Nouvelle Sér. A. 1987. V. 2. P. 1-62. [3] Beilinson A., Bernstein J. // Advances in Soviet Math. 1993. V. 16. № 1. P. 1-50. [4] Vaintrob A. On homological vector fields: Preprint. [5] Vaintrob A. Normal forms of homological vector fields // J. Math. Sciences. (to appear). [6] Courant T. // Trans. Amer. Math. Soc. 1990. V. 319. P. 631-661. [7] Alameida R., Kumpeira A. // Ann. Acad. Brasil. Ciênc. 1981. V. 53. P. 247-250. [8] Higgins Ph., Mackenzie K. // J. Algebra. 1990. V. 129. P. 194-230. 